\section{Comentario al artículo "Volumen de grasa visceral como indicador de obesidad en hombres adultos"}

\section{Commentary to the article: Visceral fat volume as an obesity indicator in adult men}

\section{Sr. Editor:}

Leímos con gran interés el reciente reporte de García Al et al. ${ }^{1}$, donde se describe la prevalencia de obesidad visceral y su relación con diversas variables clínicas y bioquímicas incluso en un contexto multivariante, demostrando algún grado de asociación entre la obesidad central y diferentes factores de riesgo cardiovascular.

Sin embargo, observamos que la ecuación empleada para estimar el "volumen de grasa visceral" es la propuesta por Amato et al. ${ }^{2}$, para el cálculo del Índice de Adiposidad Visceral (VAl), específicamente en el sexo masculino. Tras aplicar la misma, los autores clasificaron la variable según terciles obteniendo las categorías: (T1: 25,9-59,2), (T2: 59,3-77), (T3: 77,1 o más).

En relación con estos resultados, nos llaman la atención los altos valores obtenidos del VAl ("volumen de grasa visceral'") durante el proceso de clasificación. Aunque lamentablemente los autores no reportan el valor promedio de esta variable en la muestra estudiada, inferimos que se sitúa por encima de 60 , con base en las características generales observadas.

Estos resultados difieren de forma importante a los mostrados por Amato et al. ${ }^{2}$, en su estudio original donde la clasificación empleada fue por quintiles y cuyos valores más elevados fueron aproximadamente 10 (mediana del quintil $5=2,67$ ). Asimismo, Shuster et al. ${ }^{3}$, evaluó el índice en una población de 444 estudiantes brasileños cuyo valor promedio fue $1,15 \pm 0,66$, mientras que Bozorgmanesh et al. ${ }^{4}$, en el marco del Tehran Lipid and Glucose Study analizaron la influencia del VAl en la aparición de diabetes mellitus, observando un valor promedio un poco más elevado: 3,06 (95\% IC 2,99-3,13).
Si bien coincidimos en que el VAl constituye una interesante herramienta para valorar el grado de adiposidad visceral de un sujeto, especialmente en poblaciones con una prevalencia tan elevada de obesidad como ocurre en Latinoamérica $^{5}$, es importante que los autores reevalúen y esclarezcan las causas de estas importantes diferencias entre los valores obtenidos en la población colombiana y los reportes mundiales previos considerando los pocos estudios relacionados publicados hasta la fecha, especialmente en nuestro continente.

Aclarar este punto permitirá dilucidar en futuros estudios, si existen variaciones regionales tan marcadas.

\section{Bibliografía}

1. García Al, Niño-Silva L, González-Ruiz K, Ramírez-Vélez R. Volumen de grasa visceral como indicador de obesidad en hombres adultos. Rev Col Cardiol. 2016;23:313-20.

2. Amato MC, Giordano C, Galia M, Criscimanna A, Vitabile S, Midiri $M$, et al. Visceral Adiposity Index: a reliable indicator of visceral fat function associated with cardiometabolic risk. Diabetes Care. 2010;33:920-2.

3. Schuster J, Vogel P, Eckhardt C, Morelo SD. Applicability of the visceral adiposity index (VAI) in predicting components of metabolic syndrome in young adults. Nutr Hosp. 2014;30:806-12.

4. Bozorgmanesh M, Hadaegh F, Azizi F. Predictive performance of the visceral adiposity index for a visceral adiposity-related risk: type 2 diabetes. Lipids Health Dis. 2011;10:88.

5. Miranda JJ, Herrera VM, Chirinos JA, Gómez LF, Perel P, Pichardo $\mathrm{R}$, et al. Major cardiovascular risk factors in Latin America: a comparison with the United States. The Latin American Consortium of Studies in Obesity (LASO). PLoS One. 2013;8:e54056.

Juan Salazar ${ }^{\mathrm{a}, *}$, Joselyn Rojas ${ }^{\mathrm{b}}$ y Valmore Bermúdez ${ }^{\mathrm{a}}$

a Centro de Investigaciones Endocrino Metabólicas "Dr. Félix Gómez', Universidad del Zulia, Maracaibo, Venezuela

${ }^{\mathrm{b}}$ Division of Pulmonary and Critical Care Medicine, Brigham and Women's Hospital and Harvard Medical School, Boston, USA

* Autor para correspondencia.

Correo electrónico: juanjsv18@hotmail.com (J. Salazar). 